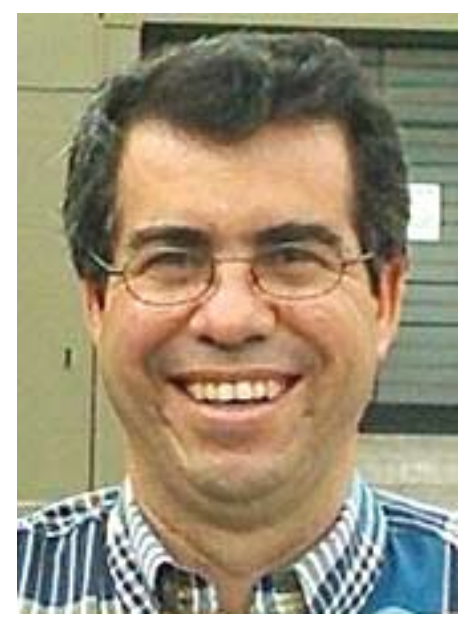

Lo que intento decir en estas páginas es que la Mater et Magistra de Juan XXIII nos enseña un método de lectura de la Doctrina Social de la Iglesia que me parece el más apropiado para hacer del magisterio social de nuestra Iglesia un aliado en nuestro caminar, como cristianos y cristianas comprometidos con una sociedad más justa. No puede la Iglesia ser "madre y maestra" si, en dialogo esotérico con su propio lenguaje, es incapaz de hablar significativamente a los seres humanos del tiempo presente.

VENTANAS ABIERTAS A LA PEDAGOGÍA UNIVERSITARIA

\title{
LEER LA MATER ET MAGISTRA CON LA MATER ET MAGISTRA
}

Pablo Mella, s. j.*

"Leer la Mater et Magistra con la Mater et Magistra" suena a pleonasmo, pero no es así. Apunta directamente a un aspecto fundamental que nos enseña esta Encíclica, y quién sabe si podríamos decir que allí reside su aporte fundamental para la historia de la Doctrina Social de la Iglesia (DOSOI).

La Mater et Magistra (MM) de Juan XXIII nos enseña un método de lectura de la DOSOI que me parece el más apropiado para hacer del magisterio social de nuestra Iglesia un aliado en nuestro caminar, como cristianos y cristianas comprometidos con una sociedad más justa. También un aliado para poder hablar, desde nuestra praxis de fe, con otras personas que quieran comprometerse en serio con la transformación de la sociedad en que vivimos.

Lo que intento en estas páginas es sugerir cómo se debe leer la Encíclica misma de acuerdo a ese método. Eso quiere decir "leer la Mater et Magistra con la Mater et Magistra".En realidad, viene a ser lo mismo que decir cómo se debe leer todo el magisterio social de la Iglesia.

Vamos a dividir este artículo-ejercicio siguiendo paso a paso la manera en que se desglosa ese método de acuerdo a los números 46 a 50 de $\mathrm{MM}$. Este análisis nos llevará directamente a las reflexiones conclusivas.

\section{Primer paso: referencia al corpus completo de la Doctrina social, en perspectiva histórica (46)'}

No se puede leer una Encíclica social sin hacer referencia a los demás documentos del magisterio social de la Iglesia. Pero al mismo tiempo, esta referencia no puede hacerse de manera a-temporal, como si nada hubiese pasado en la historia entre la publicación de un documento y de otro. En ese sentido, Juan XXIII nos enseña a leer la DOSOI en perspectiva histórica, tomando en cuenta la temporalidad del discurso que se plasma en cada documento. Las grandes encíclicas sociales se pronuncian sobre "temas del tiempo" en que fueron publicadas y llevan dentro de sí las marcas de su contexto. No profieren verdades atemporales que deberán ser interpretadas y aplicadas de la misma manera para siempre. Se trata de un magisterio "humilde", que asume la condición pasajera de su discursividad. Sin embargo, ellas siempre se escriben "conmemorando la "Rerum novarum" (7), que la MM califica como "prueba insigne" de que la Iglesia está seriamente preocupada por las cuestiones de este mundo. Es decir, al realizar un juicio contextual, el magisterio social se arriesga y asume la propia contingencia. Por esto, no se puede tildar este ejercicio de simple relativismo. Sería absurdo pensar una epistemología relativista dentro de nuestra Iglesia; pero sería obcecado creer que en la Iglesia no exista la posibilidad de asumir el propio contexto, así como los límites de las propias reflexiones normativas en temas sociales. En definitiva, se trata de tomarse en serio las cuestiones de este mundo, a pesar de su condición efímera, empeñando la propia palabra, las propias creencias y la propia vida. Con esta actitud, el magisterio social nos dice que la eternidad, lo definitivo, la comunión de Dios, está presente en la historia haciendo

"Sacerdote jesuita. Doctor en Filosofía Política, Universidad Católica de Lovaina, Bélgica. Director del Instituto Filosófico Pedro Francisco Bonó y editor de la revista Estudios Sociales.

'Los números entre paréntesis indican los párrafos correspondientes de la Encíclica, numerados de la misma forma en todas las ediciones completas. 
trascender desde dentro losmismosacontecimientos sociales, en su delimitada concreción, sin identificarse miméticamente con ellos. 0 dicho más claramente, en un contexto como el que primaba en tiempos de la Guerra Fría, el magisterio social de la Iglesia siempre pensó (y sigue pensando) que ni el capitalismo ni el socialismo son el Reino de Dios; pero que Dios está actuando en ambos, haciendo trascender la situación social hacia relaciones de mayor justicia.

Podemos concluir, por tanto, que la enseñanza magisterial de la Iglesia en asuntos sociales es histórica en tres sentidos:

a) porque remite al cristiano a la historia, a los avatares del tiempo en que vive

b) porque se pronuncia, consonantemente con lo anterior, sobre los problemas acuciantes del momento; no se pronuncia como una palabra atemporal, para todos los tiempos; es una palabra "empeñada"

c) porque reescribe constantemente su propia historia a partir de reinterpretaciones o puestas al día de la Rerum novarum, fecha en que se inicia la solicitud de la Iglesia por la "cuestión social"

Una palabra más sobre este punto c, para cerrar este primer paso metodológico de la Doctrina. Los temas abordados por la DOSOI se desarrollan dentro del esquema que Paul Ricoeur denomina "identidad narrativa". Los temas se autocomprenden dentro de una tradición escrita que arranca con la Rerum novarum, es decir, ocupándose siempre de la "cuestión social"; pero al mismo tiempo, se siente la necesidad de reescribir esa escritura previa, para poder alcanzar una comprensión más cabal (y actual) de su acción en el tiempo que les toca vivir. De esta suerte, no hay DOSOI sin hermenéutica, o lo que es lo mismo, no hay DOSOI si simplemente se repiten los temas abordados por las anteriores encíclicas de manera fundamentalista, como si la historia se hubiese detenido. La mera repetición bloqueará la posibilidad de que el amor cristiano pueda amar en los nuevos contextos de justicia planteados por la cambiante "cuestión social."

\section{Segundo paso: análisis del contexto histórico (47-49)}

El segundo paso es consecuencia lógica del primero. Si se admite que el magisterio social de la Iglesia está marcado por el contexto histórico, cuando la Iglesia se pronuncia sobre el mismo debe hacer un esfuerzo por analizar el contexto social sobre el que quiere ofrecer una guía para la acción. Esta llamada a la contextualización alcanzó a muchos grupos eclesiales a través del método adoptado por la Juventud Obrera Católica, con el famoso "ver, juzgar, actuar", ideado por el sacerdote belga, Monseñor Cardijn. El magisterio social la asume a su manera en este número de la Encíclica. Todos los grandes documentos emanados de la Conferencia del Episcopado Latinoamericano (CELAM), incluyendo el reciente de Aparecida, siguen ese esquema.

La MM analiza la sociedad contemporánea atendiendo a tres aspectos fundamentales: primero, el campo científico, técnico y económico; segundo, el campo social mundializado y tercero, el campo político

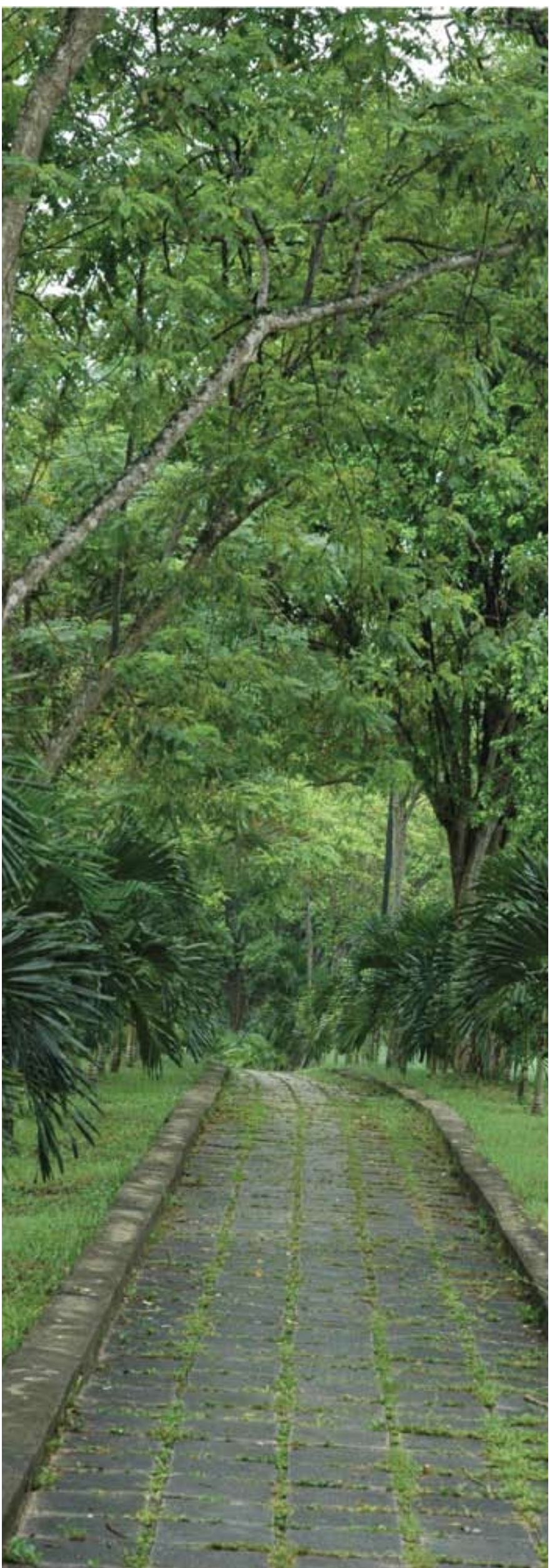




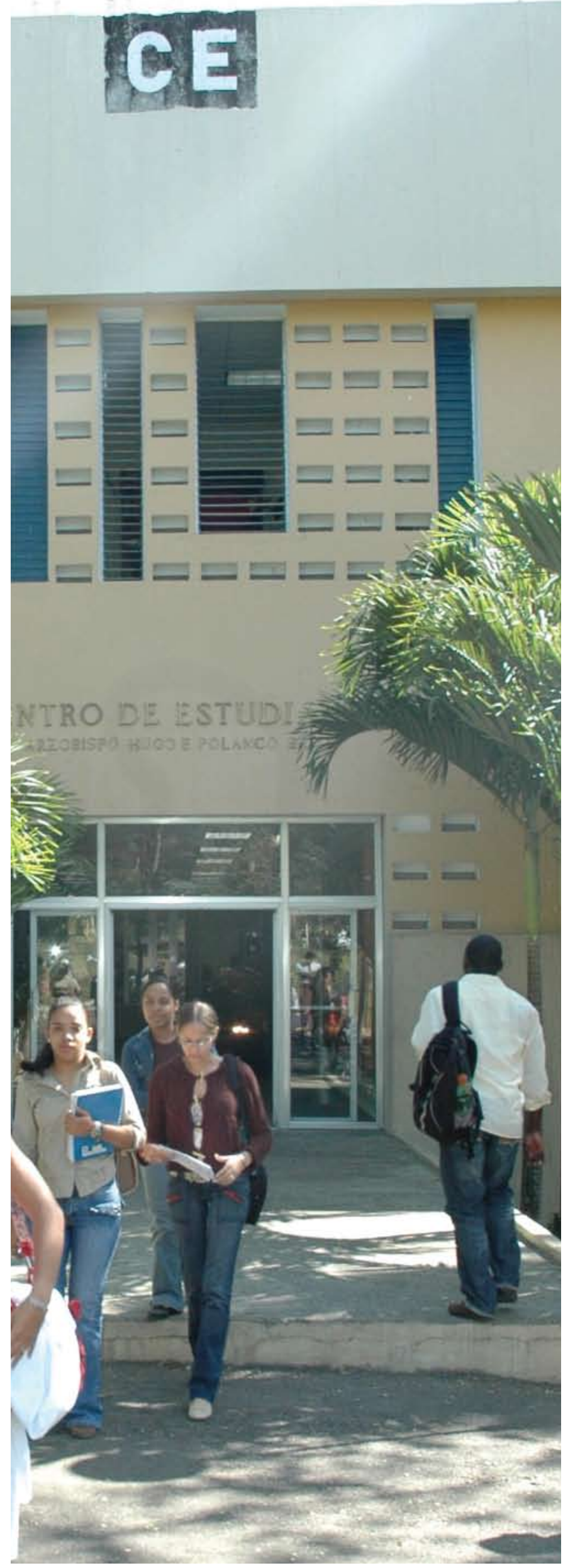

Cabe resaltar algunos puntos en este esquema. Primero, el hecho de que se comience con el análisis de la ciencia y la técnica y, además, que el campo de la economía esté integrado a la ciencia y la técnica. Ciertamente, la economía de hoy día se mueve en estrecha vinculación con el desarrollo técnicocientífico y ha tenido como resultado una mayor interconexión a escala planetaria. Es verdad que en las décadas posteriores a la MM, el capital financiero ha cobrado mucha importancia, creando las famosas burbujas económicas que no tienen sustento en la producción de riqueza material. Sin embargo, la crisis económica más reciente a escala mundial asocia el capital financiero con la biotecnología. Ante la crisis del petróleo, que explica, entre otras cosas, la Guerra de Irak y el terror antiterrorista auspiciado por Estados Unidos, buena parte de las inversiones (y de la especulación financiera) está trasladándose hacia la producción de combustibles a partir de granos o de caña de azúcar. En parte, el reciente grito de alerta contra la crisis de alimentos puede estar asociado a esta transformación de las inversiones en la economía global. Cabe referir también el incremento exponencial de las telecomunicaciones, que asocian tecnología informática de punta y la necesidad creciente de tener un mundo interconectado a escala planetaria. Buena parte del número 47 de la MM funciona casi como una profecía de lo que se ha venido a agudizar en el curso de los años.

Este dinamismo de la tecnociencia, asociado a los intereses económicos, constituye un punto de partida para entender el momento histórico que nos toca vivir. La Encíclica sugiere, por tanto, que el análisis del campo social no puede disociarse del modo en que se reorganiza el capital poniendo a su servicio el avance tecnocientífico. Además, nos recuerda que el mismo dinamismo tecnocientífico hace que el crecimiento económico de algunos países tenga como consecuencia mayores desequilibrios sociales tanto a escala nacional y regional como planetaria. De esta manera, la Encíclica despoja este crecimiento de su carácter mesiánico, auspiciado por la ideología del progreso.

Por eso, la esfera política aparecerá en estrecha relación con las transformaciones anteriores. No se le negará a lo político una cierta autonomía en relación a las otras esferas. Los deseos de autodeterminación, de libertad, de igualdad, de participación y tratamiento justo no se explican directamente desde la infraestructura económica, como hacía el marxismo. Sin embargo, las luchas específicamente políticas no aparecen desvinculadas de las transformaciones tecnocientíficas, económicas y sociales. La Encíclica llama la atención sobre la formación de instancias supra-nacionales como respuesta a los nuevos desafíos que se plantean a la humanidad como un todo; es decir, llama la atención sobre el hecho que la acción política alcanza un carácter realmente mundial.

\section{Especificación y renovación de los motivos de la acción social (50)}

Elresultadodelejerciciodediagnósticoeslacapacidaddeempatar con los motivos, ocultos o no, que realmente se encuentran en la base de nuestra acción como colectivo social. Una doctrina social 
insensible a lo que se vive en el contexto histórico de su auditorio sería una pieza de museo que incumpliría, en su mismo movimiento de salida, con el objetivo que se ha trazado.

Juan XXII lo dice solemne y poéticamente, hablando de los motivos por los cuales escribe la Encíclica: “Nos, por tanto, a la vista de lo anteriormente expuesto, sentimos el deber de mantener encendida la antorcha levantada por nuestros grandes predecesores y de exhortar a todos a que acepten como luz y estímulo las enseñanzas de sus encíclicas, para resolver la cuestión social por los caminos más ajustados a las circunstancias de nuestro tiempo". Para el Papa, MM se justifica no sólo para conmemorar la Rerum novarum, sino también "para que, de acuerdo con los cambios de la época, subrayemos y aclaremos con mayor detalle, por una parte, las enseñanzas de nuestros predecesores, y, por otra, expongamos con claridad el pensamiento de la Iglesia sobre los nuevos y más importantes problemas del momento".

Esta cita nos guiará para realizar algunas reflexiones conclusivas para nosotros, que padecemos, tanto en la Iglesia como fuera de ella, de entusiasmo por la cuestión social.

\section{Reflexiones finales}

El primer punto que nos lleva a concluir Juan XXIII es la necesidad de "mantener encendida la antorcha" de la cuestión social. Los temas sociales cansan, porque exigen demasiada abnegación. Nadie ha dicho que la búsqueda del "bien común" sea más evidente que la búsqueda del bien privado.

El segundo punto es la llamada a respetar o vislumbrar "los caminos más ajustados a la circunstancia de nuestro tiempo".
Ciertamente, el compromiso social de la Iglesia necesita de ajustes, de respeto a los caminos históricos. La Iglesia no puede cerrarse a novedades históricas, como la emergencia de nuevas figuras, de nuevos movimientos sociales o de demandas que superan "la defensa de la nacionalidad". Hoy en día, un nacionalismo poco discernido puede llevar al irrespeto de la dignidad de muchas personas que se ven forzadas a migrar de su país de origen, como explicó Benedicto XVI en su reciente visita a las Naciones Unidas. De paso, se puede perder ese motivo tan bíblico como es el reconocimiento del derecho del extranjero. Este segundo punto, puede plantearse también así: un compromiso social que no sepa acompañar los cambios históricos, no tendrá ningún motivo para existir y no podrá motivar realmente a los fieles cristianos: mucho menos "a las personas de buena voluntad" que no sean cristianas.

En tercer lugar, está la demanda de "aclarar con detalle" y "exponer con claridad". Un discurso social pertinente no puede caer en generalidades. Tiene que proceder a analizar con paciencia, haciendo distinciones, matizando y señalando lo nuevo. El resto de la MM se organiza justamente de esa manera. Este esfuerzo analítico es un signo de respeto por la misma historia y los actores históricos. El refugio en grandes declaraciones abstractas, moralizantes, está llamado a caer en el vacío. Frases como "este mundo perdido", "estos avances pecaminosos" no tienen sentido ni provocarán el compromiso social sino vienen acompañado de análisis explicativos de la situación. En fin, una vez realizado el análisis, todo debe ser "expuesto con claridad". No puede la Iglesia ser "madre y maestra" si, en diálogo esotérico con su propio lenguaje, es incapaz de hablar significativamente a los seres humanos del tiempo presente.

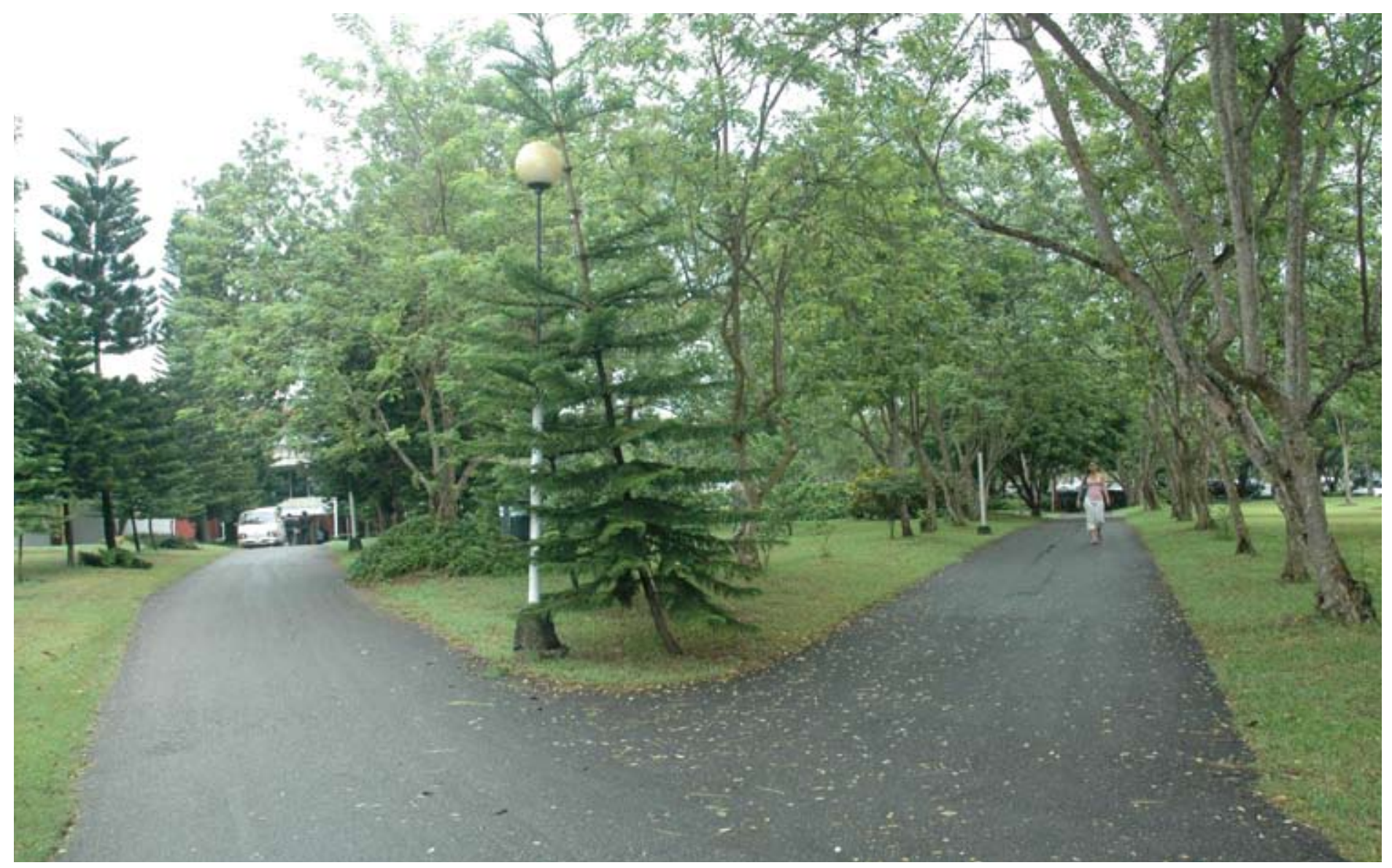

